\title{
Cell-specific translational profiling in acute kidney injury
}

\author{
Jing Liu,1,2,3 A. Michaela Krautzberger, ${ }^{1,2,3}$ Shannan H. Sui, ${ }^{4}$ Oliver M. Hofmann, ${ }^{4}$ \\ Ying Chen, ${ }^{1}$ Manfred Baetscher, ${ }^{1}$ Ivica Grgic, ${ }^{5,6}$ Sanjeev Kumar, ${ }^{1,2,3}$ \\ Benjamin Humphreys, ${ }^{5}$ Winston A. Hide, ${ }^{4}$ and Andrew P. McMahon $1,2,3,7,8$
}

\begin{abstract}
1Department of Molecular and Cellular Biology and '2Department of Stem Cell and Regenerative Biology, Harvard University, Cambridge, Massachusetts, USA. ${ }^{3}$ Department of Stem Cell Biology and Regenerative Medicine, Keck School of Medicine of the University of Southern California, Los Angeles, California, USA. ${ }^{4}$ Department of Biostatistics, Harvard School of Public Health, Harvard University, Boston, Massachusetts, USA.

${ }^{5}$ Renal Division, Department of Medicine, Brigham and Women's Hospital, Harvard Medical School, Boston, Massachusetts, USA.

${ }^{6}$ Department of Internal Medicine and Nephrology, Philipps University, Marburg, Germany. ${ }^{7}$ Harvard Stem Cell Institute, Harvard University,

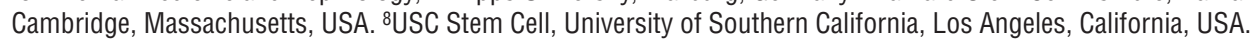

\begin{abstract}
Acute kidney injury (AKI) promotes an abrupt loss of kidney function that results in substantial morbidity and mortality. Considerable effort has gone toward identification of diagnostic biomarkers and analysis of AKI-associated molecular events; however, most studies have adopted organ-wide approaches and have not elucidated the interplay among different cell types involved in AKI pathophysiology. To better characterize AKI-associated molecular and cellular events, we developed a mouse line that enables the identification of translational profiles in specific cell types. This strategy relies on CRE recombinase-dependent activation of an EGFP-tagged L10a ribosomal protein subunit, which allows translating ribosome affinity purification (TRAP) of mRNA populations in CRE-expressing cells. Combining this mouse line with cell type-specific CRE-driver lines, we identified distinct cellular responses in an ischemia reperfusion injury (IRI) model of AKI. Twenty-four hours following IRI, distinct translational signatures were identified in the nephron, kidney interstitial cell populations, vascular endothelium, and macrophages/monocytes. Furthermore, TRAP captured known IRI-associated markers, validating this approach. Biological function annotation, canonical pathway analysis, and in situ analysis of identified response genes provided insight into cell-specific injury signatures. Our study provides a deep, cell-based view of early injury-associated molecular events in AKI and documents a versatile, genetic tool to monitor cell-specific and temporal-specific biological processes in disease modeling.
\end{abstract}

\section{Introduction}

Acute kidney injury (AKI) is characterized by a sudden deterioration in renal function associated with high morbidity and mortality (1). From $5 \%$ to $7 \%$ of hospitalized patients are reported to experience AKI $(2,3)$; the estimated annual health care expenditures attributable to hospital-acquired AKI exceed $\$ 10$ billion (4). Renal ischemia reperfusion injury (IRI) associated with renal transplantation, postcardiopulmonary bypass surgeries, and other major vascular surgeries is the leading cause of AKI; sepsis and nephrotoxic drug injury are other major contributory factors $(5,6)$. The physiological and cellular hallmarks of IRI are the loss of renal function, vasoconstriction, cell death within the renal tubular epithelium, and initiation of an inflammatory response. AKI is detected clinically by a rise in serum creatinine reflecting loss of glomerular filtration (7). Renal function can be recovered through an intratubular repair process (8), but even patients who make an apparently full recovery are at substantially increased risk of developing chronic kidney disease (CKD) (9).

To date, broad molecular characterization of AKI models has largely focused on transcriptional profiling of organ-wide signatures (10-15). Several promising biomarkers have emerged from these

Authorship note: Jing Liu and A. Michaela Krautzberger contributed equally to this work.

Conflict of interest: Research on kidney injury in the McMahon and Humphreys laboratories is supported by Evotec International.

Citation for this article: J Clin Invest. 2014;124(3):1242-1254. doi:10.1172/JCI72126. studies $(16,17)$. Analysis of temporal and spatial differences in their expression highlights the cellular complexity of the AKI response (ref. 18 and see Supplemental Figure 3; supplemental material available online with this article; doi:10.1172/JCI72126DS1), although the cellular diversity of the pathophysiological response and repair process has not been systematically addressed.

FACS using cell-specific markers is one approach to exploring specific cellular responses accompanying AKI; however, the need to dissociate the organ to single cells and the associated changes in gene activity confound the analysis of in vivo injury responses. Laser-capture microscopy on tissue sections enables local isolation of epithelial tissues but cannot distinguish among distinct cell types within nonepithelial populations or microepithelial networks such as nephron-associated vasculature.

Recently, translating ribosome affinity purification (TRAP) has been developed to identify cell type-specific changes in mRNA populations at the whole-organ level $(19,20)$. A driver for development of TRAP technology has been the need to obtain molecular signatures for adult neuronal types where recovery of intact cells is not possible through tissue dissociation and FACS. In TRAP, an EGFP-tagged form of the L10a ribosomal subunit (EGFP-L10a) is activated within a cell type of interest $(19,20)$. The profile of ribosome-bound mRNAs in the cell type is determined by affinity purification of ribosomes from a whole-organ lysate with anti-EGFP antibodies, followed by microarray profiling or sequencing of stripped mRNAs. The approach has an added advantage of focusing on the subset of mRNAs undergo- 
ing translation and thereby those mRNAs actively contributing to the phenotype of the cell.

The neural TRAP studies have used large BACs carrying cell type-specific regulatory information to drive an EGFP-L10a expression cassette within neural cell types of interest $(19,20)$. Recently, reports in Drosophila, Xenopus laevis, and zebrafish emphasize the utility of the approach (21-23). To facilitate a more generic use of TRAP in the mouse where over 500 distinct CRE recombinaseproducing mouse strains enable organ-wide, temporal, and spatial control of gene activity, we targeted EGFP-L10a into the ubiquitously expressed Rosa26 locus. Activation of the targeted EGFP-L10a cassette requires CRE-mediated removal of a transcriptional block. We combined this allele with different Cre strains to examine cell type-specific signatures in an IRI model of AKI.

Distinct TRAP signatures were generated across 4 distinct cellular compartments of the mouse kidney: the nephron, vascular endothelium, interstitial pericytes and fibroblasts, and macrophages and monocytes. The results demonstrate that Cre-loxP-TRAP is a robust and efficient methodology capable of recovering highquality TRAP signatures in rare cell populations broadly dispersed within the kidney. The results predict cellular sources of discrete components of the injury response, furthering an understanding of ischemia-associated AKI. The strategy opens the door for a comprehensive analysis of kidney injury and kidney disease and lends itself to a systematic analysis of disease states in other organ systems.

\section{Results}

Generation of a conditional EGFP-L10a mouse line. To generate a mouse line that would enable broad, cell type-specific production of an EGFP-L10a fusion protein, we targeted a construct containing the CAGGS promoter driving EGFP-L10a, with a loxP-site flanked triple SV40 polyA cassette between promoter and EGFP-L10a cassette, into the ubiquitously active Rosa26 locus. The upstream polyA cassette is designed to block activity of the downstream EGFP-L10a cassette. CRE-dependent removal of this transcriptional block activates EGFP-L10a production within the CRE-producing cell and all of its descendants (Figure 1). Details of the cloning strategy and validation and characterization of the targeting event can be found in Supplemental Figure 1, A and B, and Methods. Mice carrying the conditional EGFP-L10a allele, hereafter referred to as $L 10 a$, were maintained in a homozygous state.

Tissue-specific expression of EGFP-L10a upon Cre activation. L10a mice were crossed to $4 \mathrm{Cre}$ strains to activate EGFP-L10a expression in 4 predominantly nonoverlapping cellular compartments in the kidney. A Six2-Tet-GFP-Cre allele is active exclusively within nephron progenitors; consequently, historical labeling results in EGFP-L10a expression throughout the main body of the nephron (24). A Foxd1-GFP-Cre allele is active in the progenitors of many of the interstitial cell lineages, including those generating mesangial and nonglomerular pericytes (25). In addition, Foxd 1 is normally expressed in podocytes. $\mathrm{Cdh} 5$-Cre is reported to be active throughout the vascular endothelium (26), and finally, Lyz2-Cre specifically labels cells of the myeloid lineage, notably monocytes, macrophages, granulocytes, and dendritic cells (27). Hereafter, mice carrying any Cre allele and the $L 10 a$ allele are designated generically Cre-L10a. Six2-L10a, Foxd1-L10a, Cdb5-L10a, and Lyz2-L10a denote specifically mice that are compound heterozygotes for the indicated Cre driver and L10a.

As a first step in the analysis of these lines, we examined the cell type-specific expression of EGFP-L10a by coimmunostaining for GFP and cell type-specific markers on adult kidney sections (Figure 2). As expected, Six2-L10a kidneys showed broad labeling of the entire nephron, as evidenced by colabeling of Lotus tetragonolobus lectin-positive (LTL-positive) proximal tubules, UMOD-positive (where UMOD indicates the protein uromodulin encoded by the Umod gene) thick ascending limbs of the loop of Henle, and WT1-labeled podocytes. Foxd1-L10a kidneys displayed EGFP-L10a activity in PDGFR $\beta$-positive interstitial cells throughout the cortex and the medulla, PDGFR $\beta$-positive mesangial cells in the glomerulus,and WT1-positive podocytes. Unexpectedly, we also observed more extensive, sporadic, mosaic labeling of nephrons (Supplemental Figure 2, A and B). An independent analysis showed that this represented residual Foxd1 activity in Six2 nephron progenitors after the separation of nephron and interstitial progenitors from a common progenitor type (A. Kobayashi and A.P. McMahon, unpublished observations). Foxd1-L10a also labeled a minor subset of cells positive for the macrophage marker F4/80 (Supplemental Figure 2C). Cdh5-L10a kidneys showed GFP expression throughout the PECAM1-positive vascular endothelium, but also in a subset of F4/80-positive hematopoietic cell derivatives. Lyz2-L10a consistently and specifically colabeled F4/80-positive cells. Lyz2-L10a derivatives were exclusively seen in nonepithelial regions of the kidney, consistent with the actions of the Lyz2-myeloid arm of the hematopoietic lineage.

In summary, Six2-L10a, Foxd1-L10a, Cdh5-L10a, and Lyz2-L10a label different subgroups of cells in the kidney, consistent with the goal of identifying cell type-specific TRAP signatures. These results also predict some overlap in these signatures, in particular for podocytes and broader nephron structures shared in Six2-L10a and Foxd1-L10a populations and macrophages colabeled by Cdh5-L10 $a$ and $L y z 2-L 10 a$.

Cell-specific signatures from 4 kidney compartments through TRAP. To determine whether TRAP analysis reveals the predicted cell typespecific signatures for the Cre-L10a strains, TRAP RNA was prepared from kidneys of 6- to 12 -week-old male mice from each line and analyzed by microarray profiling. TRAP RNA yields increased in proportion to cell labeling in each strain: $10 \mathrm{ng} / \mathrm{kidney}$ for nontransgenic C57BL6/J mice, $30 \mathrm{ng} /$ kidney for L10a kidneys in the absence of CRE, $200 \mathrm{ng}$ for Lyz2-L10a, and $4 \mu \mathrm{g}$ for Six2-L10a (Supplemental Table 1). We always recovered more TRAP RNA from L10a kidneys without CRE present compared with nontransgenic controls (approximately 3 -fold), prompting a close examination of potential CRE-independent background activity of L10a. Indeed, weak EGFP transgene expression was observed in podocytes (Supplemental Figure 2D) and a subset of the aquaporin-2-positive (AQP2-positive) collecting duct epithelium (Supplemental Figure 2E), which likely reflects low-level, cell type-specific bypass of the triple SV40 polyA transcriptional stop cassette.

Following hybridization of each Cre-L10a TRAP RNA population to Affymetrix Mouse Gene 1.0 ST arrays, we performed a pairwise comparison of the normalized microarray gene expression values filtering out genes with low expression values $(<500)$. Taking the cellular overlap between Six2-L10a and Foxd1-L10a, and Cdh5-L10a and Lyz2-L10a TRAP samples into account, we analyzed this enriched data set, comparing data from each Cre-L10a sample with those of the 2 other Cre-L10a TRAP samples.

In this way, we identified 1,545 genes most specific to the nephron (Six2-L10a vs. Lyz2-L10a and Cdb5-L10a); 555 genes that are most specific to interstitial cells (Foxd1-L10a vs. Lyz2-L10a and Cdh5-L10a); 384 genes that are most endothelial specific ( $C d h 5-L 10 a$ vs. Foxd1-L10a and Six2-L10a); and 907 genes that are the most 


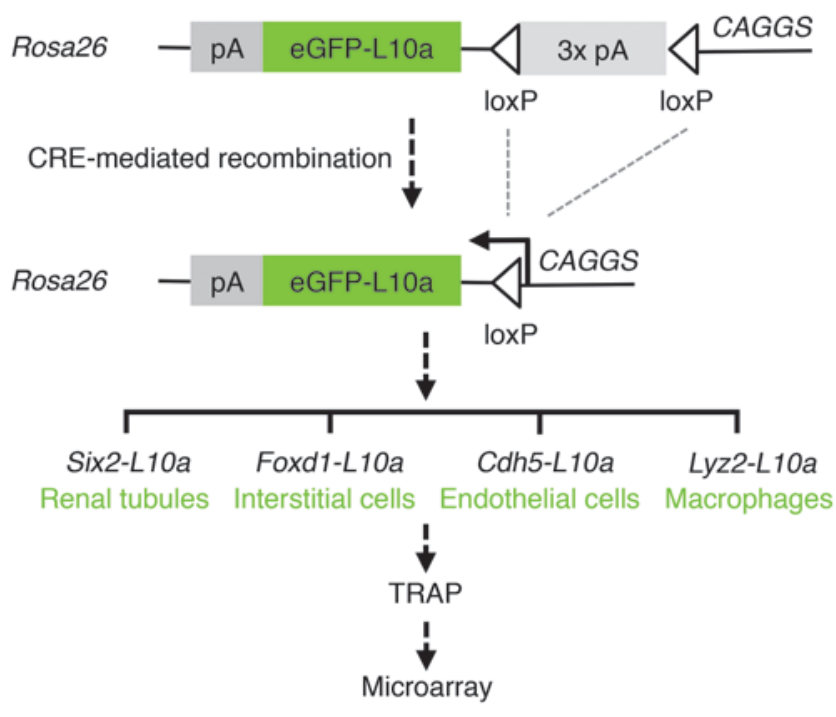

\section{Figure 1}

Schematic of Rosa26-EGFP-L10a allele and experimental work flow. The EGFP-L10a fusion protein was reversely integrated into the Rosa26 locus. Following CRE-mediated removal of the transcriptional stop cassette ( $3 \times \mathrm{pA})$, EGFP-L10a expression is driven by the CAGGS promoter. Four different Cre lines (Six2, Foxd1, Cdh5, and $L y z 2$ ) were used in this study to drive expression of the transgene in major cellular compartments of the kidney (renal tubules, interstitial cells, endothelial cells, and macrophages, respectively). RNA was isolated using TRAP prior to profiling via microarray. myeloid lineage specific (Lyz2-L10a vs. Six2-L10a and Foxd1-L10a). Data for 3 biological replicates for each TRAP RNA population are displayed in a heat map (Figure $3 \mathrm{~A}$ ). Both the predicted overlap and specific nonoverlapping signatures are evident in the heat map. In addition, we selected known markers of the main cellular types in each TRAP sample; Slc22a12, proximal tubule; Pdgfrb, interstitial cells; Flt1, vascular endothelium; Mpeg1, macrophage. We observed a strong enrichment for the expression of each gene in the expected Cre-L10a TRAP RNA sample (Figure 3B). We also observed a weaker signature for Slc22a12 in non-Six2-L10a RNA. Proximal tubule made up the majority of kidney mass and a small portion of a strongly expressed message such as Slc22a12 in proximal tubule appears to carry over in compartments with lower TRAP RNA yields (Figure 3B).

Gene Ontology (GO) biological process enrichment analysis for each of the compartment-specific gene signatures revealed a clear relationship to each of the major cell populations within each TRAP RNA sample: transport function for the nephron; cell adhesion for interstitial cells; vasculature for endothelial cells; and immune response for the macrophage-enriched myeloid population (Figure 3C). In conclusion, an accurate cell-specific mRNA signature was predicted through bioinformatics analysis of TRAP samples. The methodology is robust and reproducible, and the approach is expected to identify cell-specific TRAP RNA signatures accompanying kidney injury.

Renal IRI-responsive molecular signatures from TRAP analysis. To this end, we performed a 28-minute bilateral clamping of the renal pedicles of 6-to 12-week-old male Cre-L10a mice. As expected, serum creatinine levels were significantly elevated 24 hours after IRI, indicative of renal dysfunction in this AKI model (Supplemental Figure 3A). Kidneys from animals subjected to IRI but not sham surgery displayed typical signs of AKI: extensive renal tubular necrosis, sloughing of cells in the outer stripe of the outer medulla, the most sensitive area to the injury, and evidence of casts within tubules (Supplemental Figure 3B). RNA in situ hybridization analysis of Havcr1 (Kim-1) and Lcn2 (NGAL) expression, 2 well-characterized diagnostic markers of AKI, confirmed IRI-specific expression and highlighted the distinct cellular expression profiles for each gene (Supplemental Figure 3C). Importantly, no specific response was seen in sham-surgery controls, and the cellular pattern of Cre- $\mathrm{L1Oa}$ expression was not altered by IRI (Supplemental Figures 4 and 5).

Initially, we examined the correlation among biological replicates for no surgery and sham surgery to address the reproducibility among replicates and to rule out non-IRI-related responses to surgery. Supplemental Figure 6 highlights the correlation among biological replicates for a given Cre-L10a strain. Further, the similarity between no-surgery and sham-surgery populations indicates there is no significant impact of surgery itself on gene activity in the kidney. As predicted from the earlier reported overlap in the activity of Cre drivers, a higher correlation is observed between Six2-L10a and Foxd1-L10a, and Cdh5-L10a and Lyz2-L10a samples (Supplemental Figure 6).

Next, we applied principle component analysis (PCA), a mathematical method for finding patterns in high-dimensional data that is commonly used to highlight similarities and differences in gene expression for microarray data. PCA was applied to nosurgery, sham-surgery, and IRI TRAP profiles for all 4 Cre-L10a TRAP lines (Supplemental Figure 7). All biological replicates for a given Cre-L10a TRAP condition showed good clustering, indicative of a high degree of similarity. Further, clustering of no-surgery and sham-surgery controls was distinct from IRI experimental samples (Supplemental Figure 7). We also compared TRAP samples obtained from nontransgenic C57BL6/J kidneys, and kidneys from $\mathrm{L} 10 \mathrm{a}$ mice in the absence of CRE activity. As expected from the cellspecific read-through, we observed a markedly different grouping between these data sets (Supplemental Figure 8). Further, comparison with total RNA profiling showed that the wild-type signature reflects background isolation of RNAs in the absence of a specific TRAP product representing mRNA abundance in the whole kidney. In contrast, the major unique TRAP signatures in L10a samples reflect read-through of EGFP-L10a in principle cells of the collecting duct epithelium $\left(A q p 2^{+}\right.$and $\left.A q p 4^{+}\right)$. In summary, these data highlight the sensitivity, reproducibility, and specificity of Cre-L10a models and the markedly distinct responses made by different cell populations on IRI.

To examine cell type-specific IRI responses more directly, we compared TRAP profiling between sham-surgery and IRI samples for the 4 Cre-L1Oa lines generating 4 differentially expressed (DE) 

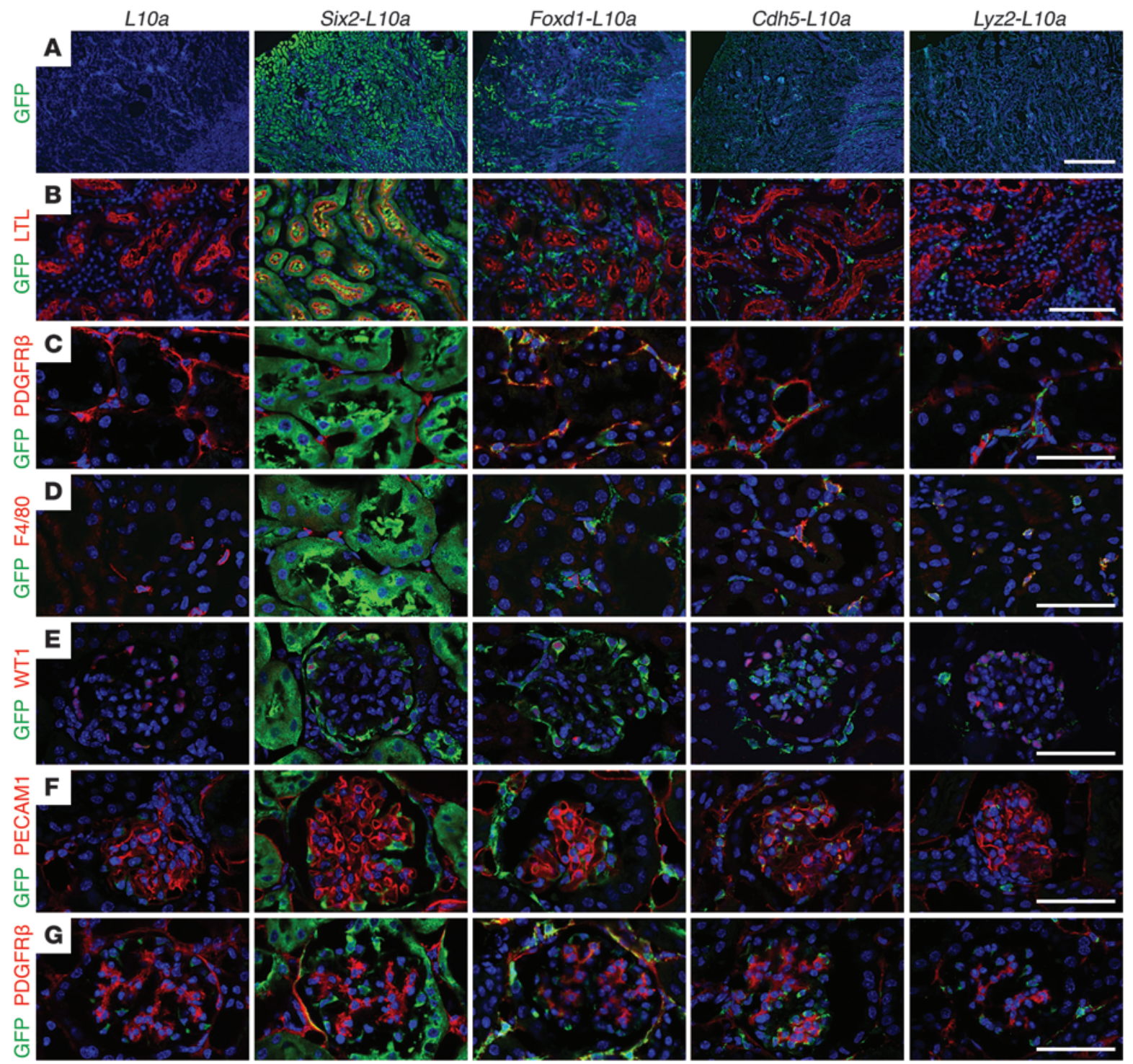

Figure 2

Double immunofluorescence analysis with GFP and cell type-specific markers in untreated kidneys of 4 different Cre- $L 10 a$ and $L 10 a$ mice. (A-G) Representative images of GFP immunostaining (green) combined with cell type-specific antibodies (red): (B) LTL for proximal tubules, (C and G) PDGFR $\beta$ for interstitial cells (C) and mesangial cells (G), (D) F4/80 for macrophages, (E) WT1 for podocytes, and (F) PECAM1 for endothelial cells. Nuclei are stained with Hoechst (blue) in all images. Scale bars: $500 \mu \mathrm{m}(\mathbf{A}), 100 \mu \mathrm{m}(\mathbf{B}), 50 \mu \mathrm{m}(\mathbf{C}-\mathbf{G})$.

gene lists with a false discovery rate (FDR) of 0.05 , a $\log _{2}$ IRI/sham ratio of greater than 1 or less than -1 , and a probe intensity value of greater than 500. DE genes meeting these criteria in each Cre population and the overlap between any 2 of these populations are tabulated in Supplemental Table 2. Ingenuity biological function comparison showed that among the most downregulated genes in the Six2-L10a and Foxd1-L10a IRI DE sets were genes associated with renal tubule activity: amino acid metabolism, small molecule biochemistry, lipid metabolism, and energy production categories, consistent with the overlap in cell labeling and the inhibition of renal tubule function in IRI. Similarly, Lyz2-L10a, but also Cdh5-L10a, showed a significant coenrichment for the upregulation of inflammatory response and immune cell trafficking genes, reflecting an overlap in L10a TRAP within the myeloid lineage
(Supplemental Figure 9). Interestingly, around 20\% of DE genes are shared among different $\mathrm{Cre}-\mathrm{L} 10 \mathrm{a}$ where there is no Cre overlap, which likely reflects common cellular responses to IRI among the different cell populations in the kidney that relate to cell survival, cell growth, and cell proliferation (Supplemental Table 2 and Supplemental Figure 9).

To better distinguish unique and shared TRAP signatures, a comprehensive heat map was generated from the merged set of 3,180 DE genes among the 4 Cre-L10a TRAP data sets compared with total RNA isolates from $L 10 a^{\text {het }}$ (the littermate control for Cre-L10a) kidneys (Figure 4A). All TRAP samples showed a significant enrichment in expression values when compared with total RNA. Further, values correlate with the fractional representation of cell types within the kidney. Whereas the strongest responses 
A

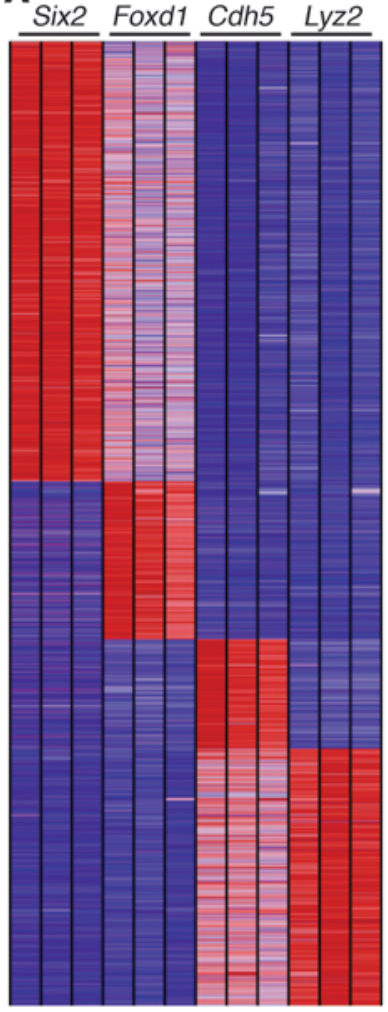

B
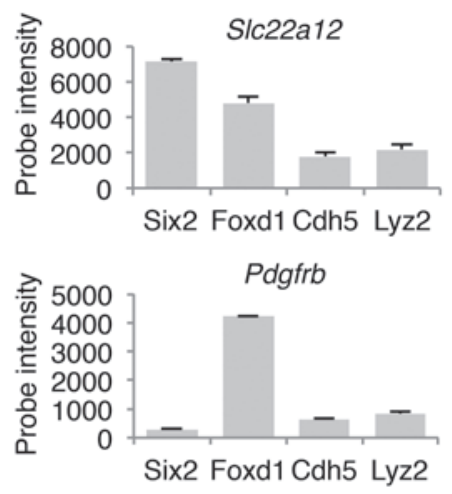

Flt1
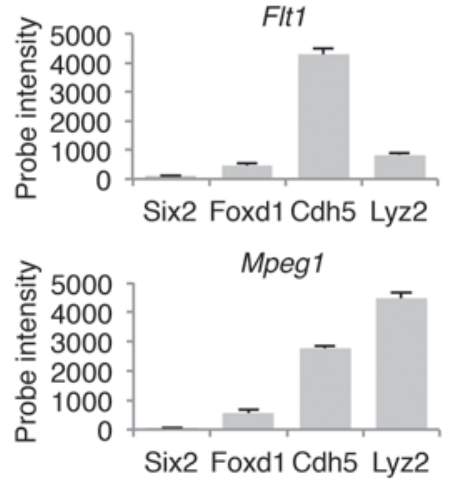

C

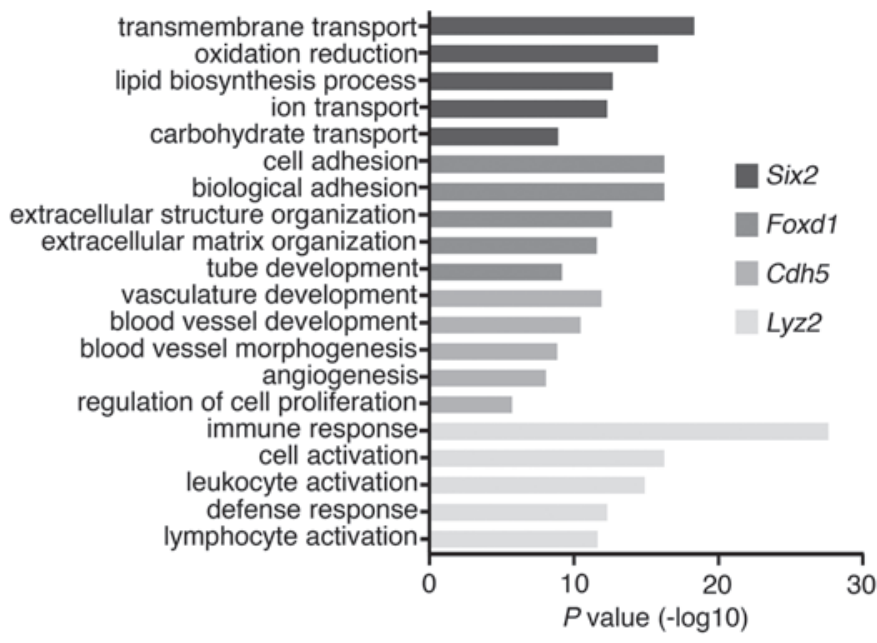

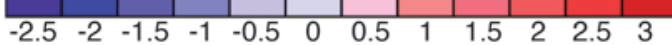

\section{Figure 3}

Cell-specific signatures and DAVID GO Biological Process analysis for 4 kidney TRAP populations. (A) Heat map showing relative gene expression value for enriched gene sets identified from 4 Cre-L10a populations (Six2, Foxd1, Cdh5, and Lyz2) of untreated kidneys. Color scale represents the relative gene expression value for each gene on each row across all 4 populations. (B) Histogram of TRAP microarray profiles (mean + SEM) across all 4 Cre-L10a populations for SIc22a12, Pdgfrb, Flt1, and Mpeg1: specific markers for nephron, interstitial cells, endothelial cells, and macrophages, respectively. (C) Top terms derived from DAVID GO Biological Process analysis for each of the 4 Cre-L10a-labeled cell populations isolated from untreated kidneys.

observed in total RNA samples correlate with Six2-L10a expression, likely reflecting the large fraction of kidney mass represented by components of the nephron, many significantly enriched TRAP RNAs were uncovered from $L y z 2-L 10 a$ kidneys where the target is a rare macrophage/monocyte contribution. The DE genes uniquely identified in TRAP samples are represented in Figure 4B. Strikingly, $88.2 \%$ of genes downregulated in the Cdh5-L10a population following IRI were exclusively identified in TRAP samples.

Independent hierarchical clustering was performed on gene sets that were up- or downregulated on IRI relative to those of shamsurgery controls (Figure 5A). This analysis highlights the cellspecific IRI responses. Interestingly, whereas most analyses of IRIinduced AKI have focused on the nephron, the largest sets of unique responses were observed in the vascular endothelium (Cdh5-L10a) and interstitial/pericyte cell populations (Foxd1-L10a), highlighting the complexity of the cellular responses and the unique insights into these events that are enabled by the TRAP approach.

GO analysis identified the top enrichment of cell-specific IRI responsive biological functions in the different cellular compartments utilizing an approach that also predicts increased or decreased activity of a highlighted biological function (Figure 5B and data not shown). Twenty-four hours after IRI, molecular transport function was predicted to be decreased in nephrons (Six2-L10a) as expected. Necrosis and apoptosis functions were also predicted to be elevated on IRI, though the analysis suggests pathway actions at 24 hours were mobilized to decrease these outcomes and to activate cell survival and cytoskeletal organization. Together, these data are consistent with damaged nephrons activating systems to block further damage and initiate repair. Analysis of the interstitial/pericyte cell compartment (Foxd1-L10a) predicts an activation of cell invasion and vasculogenesis. In myeloid lineage responses (Lyz2-L10a), the activation of cell movement, phagocytosis, and chemotaxis were consistent with the expected interplay of macrophages, monocytes, and neutrophils on IRI. Further, the recruitment of macrophages and monocytes suggested by enhanced vascular endothelial signatures $(C d b 5-L 10 a)$ points to a key role for the vasculature in regulating these populations (Figure 5B).

To examine predicted pathway activity, Ingenuity Canonical Pathway analysis was performed on the $4 \mathrm{Cre}-\mathrm{L} 10 \mathrm{a} \mathrm{DE}$ gene lists (Figure 6).

Several pathways related to decreased function of amino acids, lipid metabolism, and energy were observed in the shared Six2-L10a and Foxd1-L10a nephron signatures. Both Foxd1-L10a and Lyz2-L10a 
A

TRAP RNA

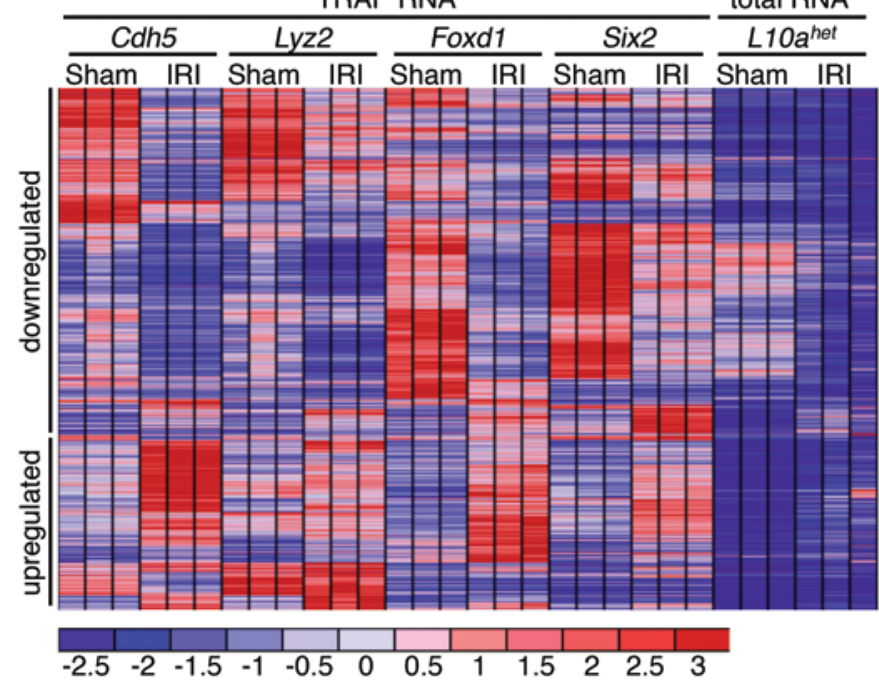

B

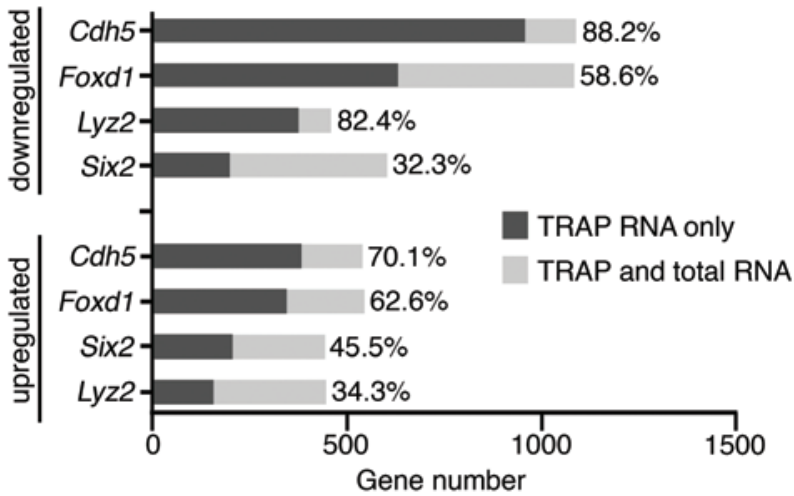

Figure 4

Cell type-specific IRI responses identified through TRAP analysis. (A) Heat map showing hierarchical clustering of 3,180 DE genes identified from at least 1 of the 4 Cre-L10a populations 24 hours after IRI compared with sham treatment. Genes with a probe intensity of more than 500 and $\log _{2}$ fold change of more than 1 or less than -1 were included. Total RNA microarray data from mice heterozygous for the L10a allele (L10ahet) are displayed for comparison. Color scale indicates relative gene expression value. (B) Comparison of the number of DE genes identified from TRAP RNA versus total RNA microarray. The percentage of genes that showed significant changes in TRAP RNA, but not in total RNA samples, are listed.

analysis predicted an LPS/IL-1-mediated inhibition of retinoic X receptor (RXR) function, while PPAR signaling was mostly enriched in Lyz2-L10a: RAR and PPAR signaling have been linked to inflammation and fibrosis in CKD (28). "Hepatic fibrosis/hepatic stellate cell" pathway terms markedly enriched in the Lyz2-L10a signature suggest shared actions of these cell types in different organ damage models. Interestingly, TRAP identifies the activation of ERK5 signaling, a key pathway that regulates tumor cell invasion and proliferation, in the Lyz2-derived cellular compartment (Figure 6). The most striking IRI feature identified from TRAP is the group of pathways specifically enriched in the Cdh5-L10a vascular endothelium, which includes CXCR4 signaling, endothelin-1 signaling, thrombopoietin signaling, and JAK/STAT signaling, emphasizing actions by kidney vasculature in response to IRI that cannot be readily recovered from microarray-based analysis of total kidney RNA samples.

Validation of cell type-specific responses to IRI treatment by RNA in situ bybridization. To confirm cell-specific predictions by visualizing the endogenous responses, we performed RNA in situ hybridization on histological sections prepared from $L 10 a^{\text {bet }}$ kidneys following sham surgery or IRI (Figure 7). For this, 1 or $2 \mathrm{Cre}-\mathrm{L1Oa}$ strain-specific candidate genes were selected for each Cre-L10a TRAP population: Cldn1 (Six2-L10a), Timp1 (Foxd-L10a), Inbbb and Foxf1a (Cdb5-L10a), and Mxd1 and Ppbp (Lyz2-L10a).

Claudin $1(C l d n 1)$ is thought to play a role is establishing new tight junctions after renal tubular cell death (29). IRI-associated activation was localized to the nephron, as predicted (Figure 7), and costaining with fluorophore-conjugated LTL antibody confirmed induction in proximal tubular epithelial cells (Figure 8A). Tissue inhibitor of metalloproteinase-1 (Timp1) is a major endogenous regulator of matrix metalloproteinase-9 (MMP-9) that plays important roles in liver IRI, fibrosis, and regeneration (30). RNA in situ hybridization revealed that its expression correlates predominantly within the interstitial cell population after IRI treatment (Figure 7).
As colabeling attempts with interstitial cell-specific PDGFR $\beta$ antibody failed due to high autofluorescence of extravasated red blood cells trapped in the interstitial compartment, we performed RNA in situ hybridization for Timp1 and Pdgfrb on consecutive sections of both sham-operated and IRI-treated kidneys. Comparison of their expression patterns demonstrated Timp1 induction in Pdgfrbpositive interstitial cells after ischemic injury (Figure $8 \mathrm{~B}$ ).

Inbbb (Inhibin $\beta$ B) encodes the $\beta$-chain of inhibin B homodimers or activin $\mathrm{BB}$ and activin $\mathrm{AB}$ heterodimers. In $b b b$ was activated broadly in peritubular cells, as shown by RNA in situ hybridization (Figure 7). Foxf1a (Forkhead box F1) encodes a transcriptional regulator, and target of the Hedgehog signaling pathway, which is activated in a CKD injury model (31). Hedgehog pathway regulation and Foxf1a activity have been linked to de novo vasculogenesis in development (32). Coexpression analysis with the vascular marker PECAM1 confirmed vascular-specific expression of both genes (Figure 9A). Thus, though Inbbb and Foxf1a expression is markedly elevated within vasculature on IRI, the 2 genes show a quite different cellular response where Foxf1a activation is confined to a few cells in the renal cortex (Figure 7).

The 2 Lyz2-L10a candidates, proplatelet basic protein (Ppbp, also known as $\mathrm{C} x \mathrm{cl} / 7)$ and max dimerization protein 1 (Mxd1), which encode a chemokine and an inhibitory component in a dimeric transcriptional complex antagonizing cMyc action (33), respectively, were both activated in cells predominantly localized to the medullary interstitial compartment (Figure 7). RNA in situ staining for $P p b p$ on consecutive sections revealed upregulation in LY-6G-labeled neutrophils after IRI treatment, while no overlap was detected with the macrophage-specific F4/80 antibody (Figure 9B). We were unable to obtain a distinct cellular confirmation for $M x d 1$ because of background issues in the immunostaining protocol, but we note that previous studies have reported induction of $M x d 1$ expression during myeloid differentiation $(34,35)$. 
A

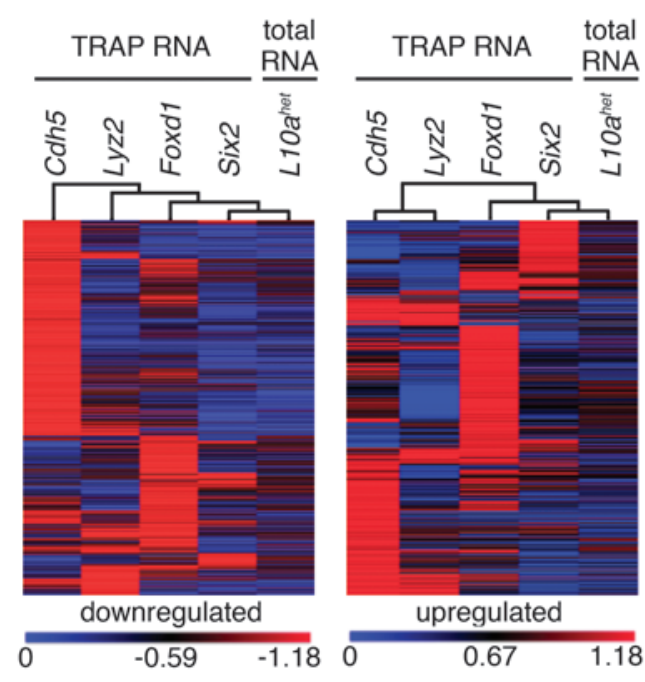

B

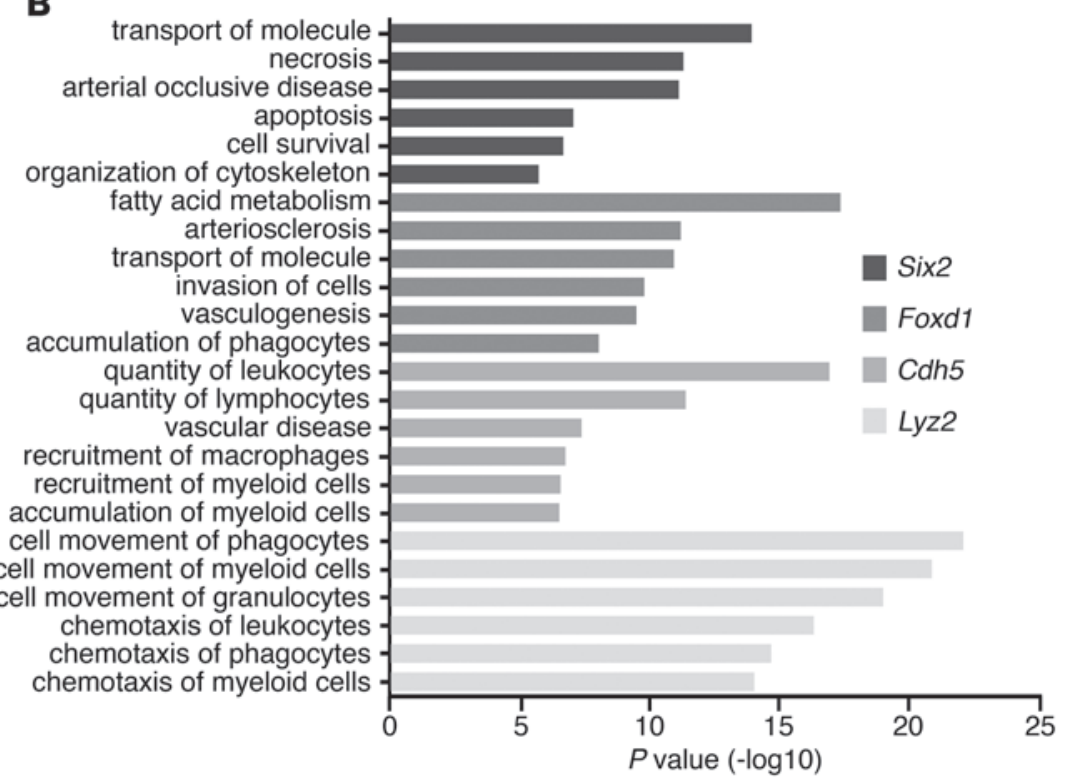

\section{Figure 5}

Hierarchical clustering and ingenuity biological function analysis of DE genes. (A) Heat maps displaying hierarchical clustering of DE genes (IRI vs. sham; left: downregulated, right: upregulated) identified from TRAP data sets (not in total RNA) from 4 Cre-L10a populations. Total RNA microarray data for mice heterozygous for the $\angle 10 a$ allele $\left(L 10 a^{h e t}\right)$ are displayed for comparison. Color scale indicates relative log ${ }_{2}$ ratio (IRI vs. sham). (B) Top terms derived from ingenuity biological functional analysis comparing DE genes (IRI vs. sham) for each of the 4 different Cre- $L 10 a$ populations.

In summary, these experiments corroborate the predictions from the TRAP analysis and, in addition, confirm cell-type specificity for at least 1 of the candidates identified from each of the 4 target populations.

\section{Discussion}

This study reports what we believe to be a novel Cre/loxP mouse strain, Rosa26-EGFP-L10a, which enables cell type-specific translational profiling in potentially any cell type in any organ system in the mouse. To illustrate the utility of the system and the power of the approach for identifying cell type-specific translational signatures, we focused on generating new insights in an IRI model of AKI. Several observations support the view that the system provides a robust, reproducible, and sensitive platform for studying cell-specific responses in kidney injury.

First, we recovered expected cell type features through bioinformatics analysis of distinct TRAP populations based on the documented cellular distribution of the CRE-activated EGFPL10a component. Second, within any TRAP population, biological replicates grouped more closely with each other than with any other population in a PCA. Third, IRI led to a markedly different profile for each Cre-L10a population, and analyses of these cellular signatures showed strong association with known or predicted features of the regulatory responses within different cell populations. Fourth, we confirmed cell-specific expression of a number of highly cell type-specific gene predictions through RNA in situ hybridization. Fifth, we observed a strong association of our strongest "hits" with combined studies of shared AKI response genes in whole-kidney data sets based on IRI, sepsis, and nephrotoxic injury insults. Of a group of 62 genes that showed 2 or more indications among 7 published studies (11), 79\% (49 genes) show a 2-fold or greater difference in the Cre-L10a TRAP data sets. This provides an independent validation of relevance of our data around the subset of genes that displays the largest expression changes detectable in total kidney analysis. Finally and most importantly, we demonstrate that even rare cellular responses, such as those in the Lyz2-L10a compartment that we estimate as less than $0.5 \%$ of the cellular content of the kidney, are captured in the TRAP approach (see data in Figures 7 and 9). The ability to discern gene expression responses in rare cell populations with a rapid, simple methodology that does not require sophisticated systems for cell isolation should greatly enhance studies of complex regulatory processes at the cellular level in vivo.

Our comprehensive TRAP data sets provide a resource for investigating AKI, for comparison between AKI and other kidney disease models, such as chronic kidney injury where AKI is a significant risk factor for CKD in later life, and for comparison of IRI in other organ systems where data indicate shared, as well as organspecific, responses to the original insult.

As demonstrated herein, biological function and pathway analysis give new insights into distinct cellular responses occurring within the damaged kidney that argue persuasively for a more comprehensive analysis of these cellular events for a better understanding of damage, repair, and disease susceptibility following AKI.

The cell types most vulnerable to IRI in the mouse kidney are cells of the S3 segment of the proximal tubule contained within the Six2-L10a population. By 24 hours after IRI, bioinformatics analysis suggests that strong antiapoptotic and antinecrotic pathway activity, cell movement, junction formation, and cell proliferation are at play, consistent with retaining viability of cells that remain within the renal tubule, maintaining a patent epithelial 


\begin{tabular}{|c|c|c|c|c|}
\hline \multirow[b]{2}{*}{ LPS/IL-1 Mediated Inhibition of RXR Function } & Six2-L10a & Foxd1-L10a & Cdh5-L10a & $L y z 2-L 10 a$ \\
\hline & $\square 4.46$ & $\mathbf{\square} 9.72$ & $=1.14$ & घ9.84 \\
\hline \multirow{2}{*}{$\begin{array}{r}\text { Folate Polyglutamylation } \\
\text { ILK Signaling }\end{array}$} & $=4.44$ & $=3.62$ & $=2.34$ & \\
\hline & $\mathbf{m} 4.39$ & & $\varpi 1.64$ & \\
\hline Folate Transformations I & $=4.37$ & $=3.39$ & $=2.43$ & \\
\hline Coagulation System & $=5.98$ & $\square 4.77$ & $=1.24$ & $=3.79$ \\
\hline Glycine Betaine Degradation & $=5.43$ & $=5.54$ & $=2.24$ & \\
\hline NAD biosynthesis II (from tryptophan) & $=4.36$ & $=4.21$ & $\varpi 0.51$ & $=0.87$ \\
\hline Tryptophan Degradation III (Eukaryotic) & $=4.33$ & $=4.81$ & $=0.67$ & $=1.20$ \\
\hline Leucine Degradation I & $=5.81$ & $\mathbf{m} 3.39$ & $=2.43$ & $=2.16$ \\
\hline Role of Tissue Factor in Cancer & $=4.22$ & $=2.31$ & $=5.84$ & $\square 4.31$ \\
\hline Glutathione-mediated Detoxification & $=4.16$ & $=5.07$ & $=2.26$ & $=4.54$ \\
\hline Superpathway of Cholesterol Biosynthesis & $=4.04$ & $=3.34$ & $=0.38$ & \\
\hline Valine Degradation & $=3.65$ & $=4.29$ & $\varpi 0.69$ & \\
\hline LXR/RXR Activation & $=3.22$ & $=5.13$ & $=1.50$ & \\
\hline Superpathway of Methionine Degradation & $=1.72$ & $=5.58$ & $=3.40$ & $=0.79$ \\
\hline Cholecystokinin/Gastrin-mediated Signaling & $=1.76$ & $=0.84$ & $=4.39$ & $=3.09$ \\
\hline Leukocyte Extravasation Signaling & $=1.60$ & $\square 1.46$ & $=4.25$ & $=3.61$ \\
\hline IL-8 Signaling & $=1.47$ & $=2.18$ & $=7.84$ & $=3.87$ \\
\hline HMGB1 Signaling & $=1.25$ & $=0.59$ & $=4.43$ & $=2.41$ \\
\hline Toll-like Receptor Signaling & $\mathbf{E 1 . 1 4}$ & $=0.47$ & $\square 6.00$ & $=2.38$ \\
\hline CXCR4 Signaling & $=0.76$ & $\square 0.29$ & $=4.22$ & $\square 1.02$ \\
\hline Endothelin Signaling & $=0.56$ & $=0.74$ & $=4.14$ & $=0.78$ \\
\hline Chemokine Signaling & $=0.53$ & $=0.27$ & $=4.07$ & $=2.89$ \\
\hline Thrombopoietin Signaling & $=0.25$ & $=0.74$ & $=4.10$ & $=1.38$ \\
\hline JAK/Stat Signaling & & $=1.62$ & $=4.23$ & $\Xi 1.91$ \\
\hline IL-10 Signaling & 2.55 & $\square_{1.23}$ & $\mathbf{m} 5.36$ & $\square 6.39$ \\
\hline Acute Phase Response Signaling & $=2.51$ & $=2.76$ & $\mathbf{m} 4.65$ & $=4.48$ \\
\hline Glucocorticoid Receptor Signaling & $=0.67$ & $=0.94$ & $=4.64$ & $\square 4.05$ \\
\hline Hepatic Fibrosis/Hepatic Stellate Cell Activation & $=2.18$ & $=4.03$ & $=2.46$ & $\varpi 8.27$ \\
\hline Aryl Hydrocarbon Receptor Signaling & $\varpi 1.50$ & $\mathbf{m} 4.91$ & $\varpi 1.82$ & $=4.62$ \\
\hline IL-6 Signaling & $=3.91$ & $=3.85$ & $=2.75$ & $\square 5.40$ \\
\hline PPAR Signaling & $\mathbf{m} 1.57$ & $=2.22$ & $=2.27$ & $\square 4.09$ \\
\hline IL-17A Signaling in Fibroblasts & $\varpi 0.96$ & $=1.15$ & $=3.52$ & $=4.63$ \\
\hline ERK5 Signaling & $=2.80$ & $\square 1.80$ & $\varpi 1.97$ & $\square 4.55$ \\
\hline Xenobiotic Metabolism Signaling & $=1.54$ & $=3.24$ & $\varpi 1.28$ & $\mathbf{\square} 4.96$ \\
\hline$r$ val & ue $(-\log 10)$ & $\mathbf{0}>8$ & $=2-4$ & $\square<2$ \\
\hline
\end{tabular}

Figure 6

Canonical pathway analysis. Unique and shared canonical pathways overrepresented within the 4 different Cre-L10a populations. Comparison of Ingenuity Canonical Pathway analysis was done using DE gene lists (IRI vs. sham) identified from the different Cre-L10a populations. neutrophils). Importantly, TRAP faithfully captured the well-known endothelial responses to hypoxia associated with maintenance of vascular integrity (angiopoietin 2 [Angpt2]) $(36,37)$ and regulation of trafficking of inflammatory cells in and out of the hypoxic tissue microenvironment (chemokine receptor 4 [Cxcr4] (38-40) and intercellular adhesion molecule-1 [Icam-1]) (41-43). A preliminary analysis of 2 TRAP targets that were markedly enriched within endothelial cells hints at the spatial diversity of endothelial response.

Consistent with the activation of myeloid lineages, we see evidence of receptor (chemokine [C-C] motif receptor 7 [Ccr1], G protein-coupled receptor; cannabinoid receptor 2 (macrophage) [Cnr2]; colony-stimulating 3 receptor (granulocyte) [Csf $3 r]$; chemokine $[\mathrm{C}-\mathrm{C}]$ motif receptor 7 [Cxcr2]; formyl peptide receptor 1 [Fpr1]) and pathway activation (IL-10 signaling) specifically within the Lyz2-L10a population, and their associated cellular actions and activities, such as phagocytosis and movement. We also see evidence for the production of signaling factors (oncostatin M [Osm], cytokine, growth factor [Tgfb3]) and for a parallel signaling response in renal epithelium supporting a direct role of macrophages in renal repair.

Vascular endothelial damage and an associated fibrotic response are linked to CKD (44). One early response of the renal endothelium to IRI is characterized by the upregulation of adhesion molecules promoting endothelial-leukocyte interactions: upregulation of Icam1, and Sele (E-selectin) was unique to Cdh5-derived TRAP data sets.

tubular network, and cell division to repair the nephron, which is thought to be largely driven through division of remaining renal tubule cells after the initial wave of cell death (8). We also see a marked downregulation of many genes that are a normal component of the essential physiology of tubular epithelial cells. This may reflect an essential change in cell properties that accompanies the first steps in repair of nephron function.

We also observe specific responses within other cell populations that may shed light on the complex pathophysiology following IRI. The endothelium, a relatively neglected component of these processes, shows a dramatic change in TRAP profile. Among the genes whose translation is markedly activated are cytokines $(C c l 2, E d n 1)$ and chemokines ( $\mathrm{C} x \mathrm{cl} 2 \mathrm{)}$ ) associated with activation, invasion, and expansion of myeloid cell types (macrophages, monocytes, and
Foxd1-derived interstitial populations also showed upregulation of genes associated with cell-cell adhesion and tissue repair following IRI. Integrin $\alpha 5$ (Itga5) is reported to have beneficial effects in nerve injury (45) and bone repair (46). RNA in situ hybridization shows marked upregulation within the interstitium (data not shown), suggesting that cell adhesion-based repair process utilizing Itga 5 may also be triggered on AKI.

As further evidence of the strength of these data sets, we observe corroborative data supporting recent insights into expanding signaling functions for important vascular regulatory factors. Flt1 encodes a VEGF receptor, classically linked to control of vascular endothelium. We observed the expected Flt1 TRAP signature enriched within the Cdh5-L10a TRAP, but also a marked signature specific to Foxd1-L10a TRAP: interstitial fibroblasts and pericytes. 
A
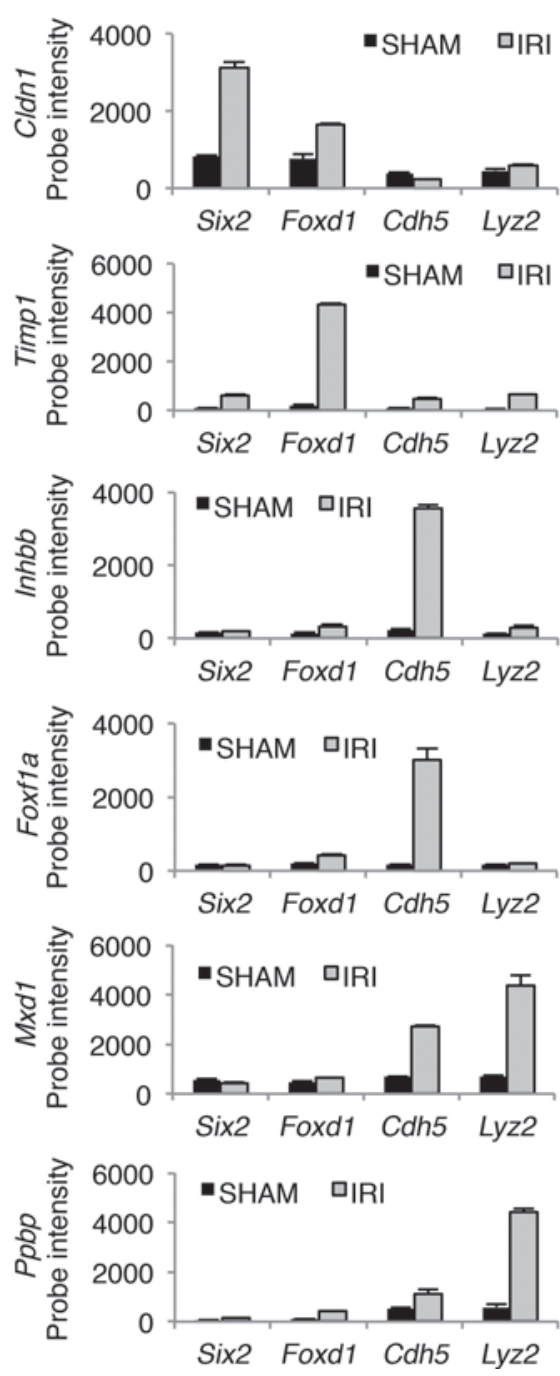

B
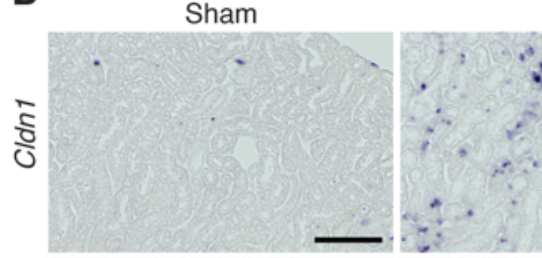

$|R|$
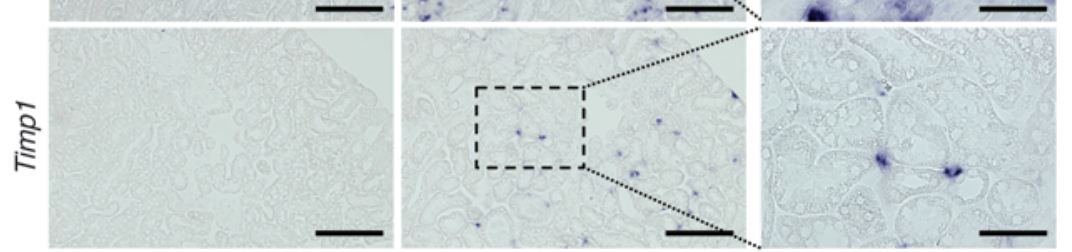

ङ
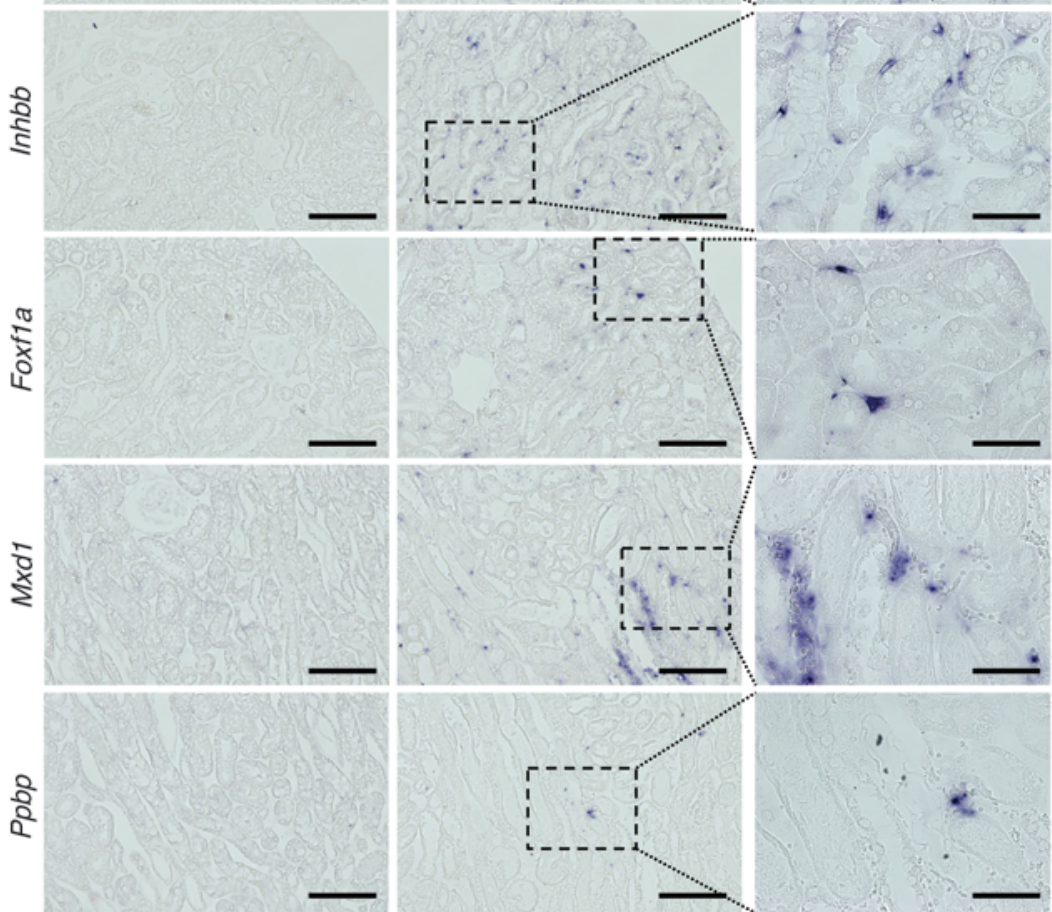

Figure 7

Visualization of expression patterns of cell type-specific IRI-responsive genes by RNA in situ hybridization. (A) Histograms of microarray probe intensities (mean + SEM) of cell type-specific IRI-responsive genes across 4 different Cre-L10a populations and (B) their respective expression patterns in sham-operated and IRI kidneys visualized by RNA in situ hybridization. Scale bars: $200 \mu \mathrm{m}$ (left and middle columns); ingenuity canonical pathway $50 \mu \mathrm{m}$ (right column).

Recent evidence demonstrates Flt1 expression in pericytes. Further, functional studies highlight the critical role for Flt1 in pericyte biology (47). Capillary rarefaction and pericytes contributes to renal fibrosis postischemic AK $(48,49)$. Treatment with VEGF-121) was found to ameliorate capillary rarefaction and fibrosis after ischemic injury (50). These intriguing observations provide a foundation for further hypothesis-driven, mechanistic-based studies to investigate the yet-unexplored role of Flt 1 in the acute and chronic pathophysiology of ischemic AKI.

Our study also highlights a role for activation of ERK5 signaling in the Lyz2-derived cellular compartment in vivo from target pathway prediction. ERK5 action underlies the CSF-1-induced proliferation of macrophages in vitro and is emerging as a key regulator of tumor cell invasion and migration (51-53). Interestingly, Csf1 is substantially elevated in the Foxd1- and Lyz2-derived cellular com- partments in our study, consistent with a role for ERK5 regulation of proliferation, migration, and invasion of Lyz2-derived cells in the setting of ischemic AKI.

The system we describe offers advantages and some inherent disadvantages, depending on the experimental goal, over BACmediated transgenic approaches for TRAP.

First, the system leverages the increasing bank of Cre-driver mice, enabling the researcher to combine any cell type-specific Cre driver with a single, well-characterized EGFP-L10a-producing strain. This negates the requirement to generate a distinct BAC-EGFP-L10a strain where a Cre line exists for a cell population of interest. However, as is evident here, there can be expected or unexpected activities of Cre strains, and the historical nature of CRE action at an earlier time point can complicate analysis. The use of temporal labeling with the rapidly expanding resource of 
A

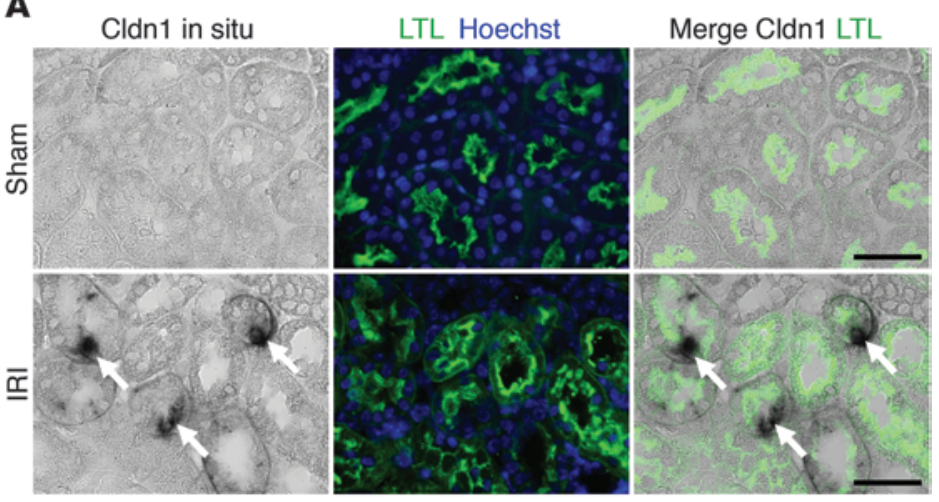

B

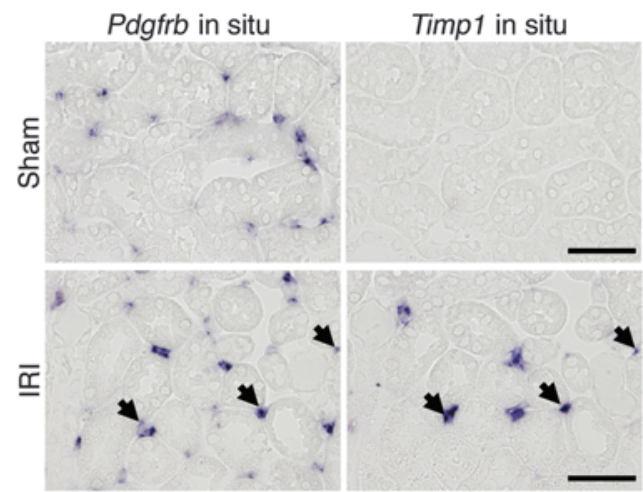

\section{Figure 8}

Validation of cell type-specific expression of IRI-responsive genes identified from Six2-L10a and Foxd1-L10a data sets. (A) Cldn1 RNA in situ hybridization coupled with immunofluorescent staining for LTL confirms induction of Cldn1 (arrows) in LTL-positive proximal tubules in IRI-treated kidneys. Nuclei are stained with Hoechst (blue; middle column). (B) RNA in situ hybridization on consecutive sections demonstrates expression of Timp1 in Pdgfrb-positive interstitial cells (arrows) after IRI treatment. Scale bars: $75 \mu \mathrm{m}$.

tamoxifen-inducible Cre-ER and Cre-ERT2 strains can overcome some of these issues. Importantly, with knowledge of potential overlap, bioinformatics approaches can be tailored to the analysis as we have demonstrated in this study. Second, the use of a single EGFP-L10a line will facilitate comparative studies across and within organ systems where each individual BAC insertion may have features of expression level or spatial activity. In this regard, though mice producing EGFP-L10a from the R26 locus-inserted CAGGS-EGFP-L10a cassette in all cells are born at a normal Mendelian ratio and are superficially indistinguishable from littermates, they fail to thrive postnatally, suggesting a negative effect of transgene activity in some postnatal cell types.

Independent of the regulatory system that drives EGFP-L10a activity, the approach obviates the need for tissue dissociation and FACS profiling to obtain gene expression signatures from distinct cell populations within an organ: a critical consideration where dissociation of living intact cells is not possible (as in many neurons with the adult nervous system) or where cell dissociation and FACS triggers damage responses that may obscure an in vivo damage-repair response as in our study. Importantly, TRAP isolates mRNAs in active translation, so the overall approach gives the best insight into ongoing protein synthesis in contrast to general isola- tion of cellular RNA. However, the approach is restricted to the mRNA component of transcribed genome.

In summary, we have documented and validated a general, widely applicable system for exploring TRAP-directed mRNA profiles in any cellular or temporal context, and in any tissue type, depending on a complementary CRE strain. We have confirmed and extended studies largely based on wholeorgan analysis. The available data provide a rich source of information that enhances our understanding of the cellular roles and cellular mechanisms of AKI. An extension of this approach to earlier and later stages will provide a comprehensive, cellular insight into damage and repair phases in AKI and the development of cell type-specific indicators of damage, repair, and postrepair disease susceptibility.

\section{Methods}

Mice. The Rosa26-EGFP-L10a allele was generated using recombination-mediated cassette exchange in F1 (C57BL6/J;129/Sv) ES cells similar to an earlier approach in the laboratory (54). Briefly, a parental ES clone (\#456B3) was generated by targeting F1 cells with a Rosa26 targeting vector containing an F3- and FRT-flanked ATG-F3-Puro-pA cassette followed by a PGK promoter driving expression of FLPo recombinase. The exchange vector contained a neomycin resistance gene (Neo) that lacks the ATG translation start codon, followed by a CAGGS promoter, a loxP-flanked triple SV40 polyA signal, and the EGFP-L10a cassette. Three copies of the 400 -bp core region of chicken $\beta$-globin insulator (55) were inserted upstream of the CAGGS promoter to block local influences on the promoter activity. Successful recombination resulted in insertion of a single copy of the CAGGS-loxP-3xpA-loxP-EGFP-L10a-pA cassette and allowed for $\mathrm{Neo}$ expression from the endogenous Rosa 26 promoter. Correct targeting was confirmed by long-range PCR. CRE-dependent expression of EGFP-L10a was examined by GFP immunostaining. We used 4 cell type-specific Cre lines to drive EGFP-L10a expression in the main renal tubule component of the nephron (Six2-derived) (McMahon lab) (56): interstitial mesenchyme, mesangial cells, pericytes and juxtaglomerular apparatus (JGA) (Foxd1-derived) (McMahon lab) (57); vascular endothelium (Cdh5/Pecam/VE-cad-derived) (JAX) (58), and macrophages (Lyz2-derived) (JAX) (59).

IRI. An established model of warm IRI was used to induce ischemic acute renal failure $(60,61)$. Briefly, 6 - to 12 -week-old male mice were anesthetized with an intraperitoneal injection of a ketamine/xylazine mix (105 mg ketamine $/ \mathrm{kg} ; 10 \mathrm{mg}$ xylazine $/ \mathrm{kg}$ ). The body temperature was maintained at $37^{\circ} \mathrm{C}$ throughout the procedure. Kidneys were exposed through flank incisions, and both renal pedicles were clamped for 28 minutes using nontraumatic microaneurysm clips (Roboz Surgical Instrument Co.). Restoration of blood flow was monitored before closing incisions with surgical staples. Sham-operated mice underwent the same procedure except for clamping of the pedicles.

TRAP and microarray. TRAP followed a detailed protocol supplied by the Dr. N. Heintz laboratory at the Rockefeller University (New York, New York, USA) $(19,20)$. Kidneys were removed 24 hours after surgery. Briefly, mice were anesthetized, perfused with ice-cold PBS, and kidneys removed; these were minced rapidly on ice, then added to ice-cold polysome extraction buffer for homogenization (4 $\mathrm{ml}$ per kidney). Monoclonal anti-GFP antibodies (Monoclonal Antibody Core Facility, Memorial Sloan-Kettering Cancer Center, New York, New York, USA) were coupled to Dynabeads and added to a postmitochondrial preparation of kidney extract at a ratio of $200 \mu \mathrm{l}$ of freshly bound beads to 800 to $1,200 \mu \mathrm{l}$ of kidney superna- 
A

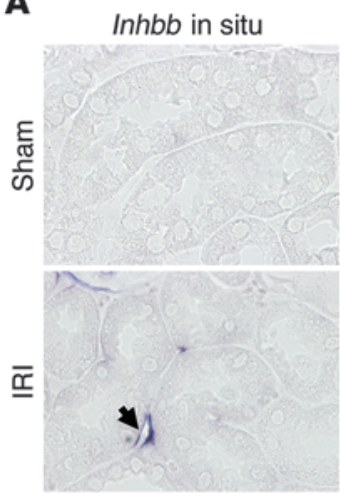

Foxf1a in situ

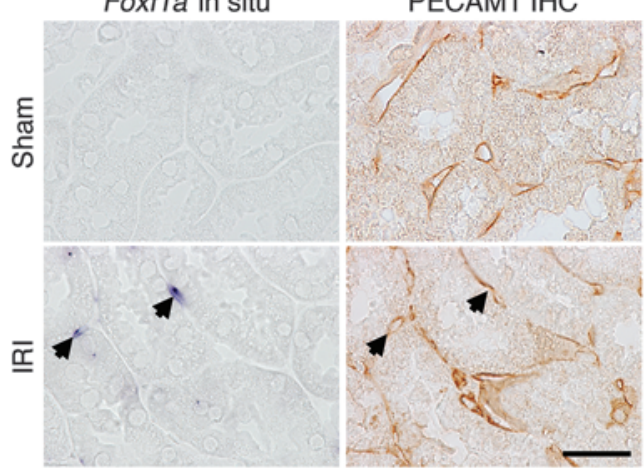

PECAM1 IHC

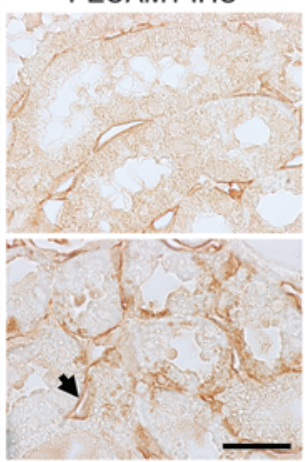

PECAM1 IHC
B

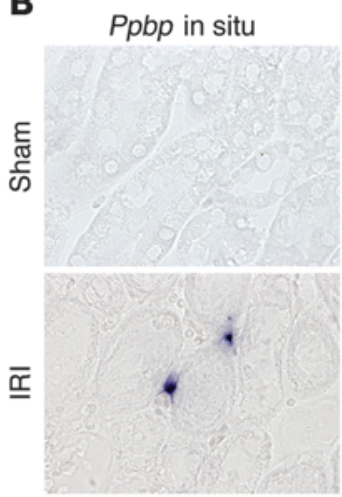

Ppbp in situ

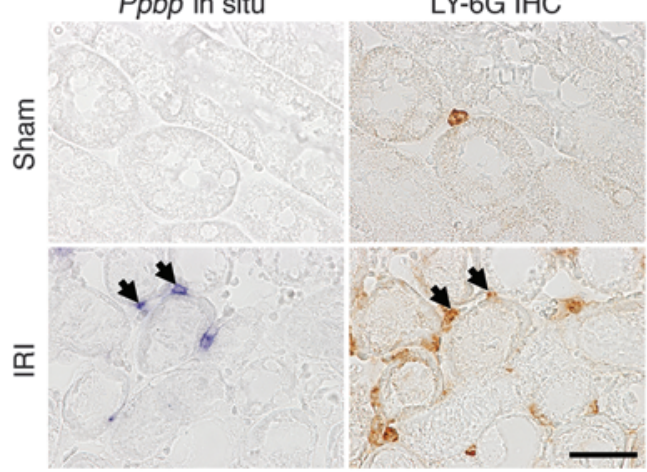

Figure 9

Validation of cell type-specific expression of IRI-responsive genes identified from Cdh5-L10a and Lyz2-L10a data sets. (A) RNA in situ hybridization for Inhbb (top) and Foxf1a (bottom) coupled with immunostaining (IHC) for PECAM1 on consecutive sections confirms induction in endothelial cells (arrows) after IRI treatment. (B) RNA in situ hybridization for Ppbp coupled with immunostaining for F4/80 (top) and LY-6G (bottom) on consecutive sections showed no overlap with the F4/80-positive macrophage population, but revealed colabeling with LY-6G antibody, a marker for monocytes and neutrophils (arrows). Scale bars: $75 \mu \mathrm{m}$.

tant. Beads and extract were incubated at $4^{\circ} \mathrm{C}$, with agitation, for $30 \mathrm{~min}$ utes. The beads were then washed through a large volume of wash buffer, resuspended in lysis buffer, and RNA isolated; contaminating DNA was removed by DNaseI digestion (QIAGEN RNAeasy Mini Kit). The integrity of the final RNA preparation was assessed using an RNA nano-chip on an Agilent 2100 Bioanalyzer; RNA yield was determined by Nanodrop 2000 (Thermo Scientific) measurement. Two hundred nanograms of purified RNA (total or EGFP-L10a purified) was used to generate cDNA, and ultimately, cRNA for expression analysis on Affymetrix Mouse Gene 1.0 ST arrays. Washing of arrays was performed using an Affymetrix GeneChip 450 Fluidics station. Scanning of arrays was performed by an Affymetrix 3000 GeneChip Scanner. Total RNA was isolated by conventional procedures (QIAGEN RNAeasy Mini Kit).

Bioinformatics analysis. Microarray data have been deposited (GEO GSE52004). Microarray data were normalized by robust multichip average (RMA) in the oligo BioConductor package, which normalizes the intensity values at the transcript level and collapses probes into "core" transcripts based on annotations provided by Affymetrix. Heat maps and hierarchical clustering were done with GenePattern (Broad Institute) and $\mathrm{MeV}$ (MultiExperiment Viewer). PCA was done using Partek Genomics Suite. GO, biology function annotation, and pathway analysis were done using DAVID Bioinformatics Tools and Ingenuity Pathway Analysis. A linear model for microarray data analysis (Limma) was performed, and pairwise comparisons were applied to identify DE genes across 4 cell populations that had a 2 -fold or greater change in expression and an FDR-corrected $P$ value of less than 0.05 . Differential expression was calculated using an empirical Bayes model that uses a moderated $t$ statistic to test the average difference in log expression levels between groups while borrowing information across genes to take individual variances into account.

Serum creatinine analysis. Serum creatinine levels were measured at the Washington University George M. O'Brien Center for Kidney Disease Research (St. Louis, Missouri, USA) using an automated blood analyzer (Cobas Mira Plus; Roche).

Histology. Kidneys were perfused with ice-cold PBS and embedded in paraffin after overnight fixation in $4 \%$ paraformaldehyde at $4{ }^{\circ} \mathrm{C}$. Sections were cut at $5 \mu \mathrm{m}$ and stained with $\mathrm{H} \& \mathrm{E}$.

Immunofluorescence. Immunofluorescent analysis was performed on 8- to $10-\mu \mathrm{m}$ frozen sections. Sections were incubated overnight at $4^{\circ} \mathrm{C}$ with primary antibodies against GFP (GFP-1020; Aves Labs), LTL (FL-1321; Vector Labs), UMOD (BT-590; Biomedical Technologies Inc.), AQP2 (sc-28629; Santa Cruz Biotechnology Inc.), WT1 (sc-192; Santa Cruz Biotechnology Inc.), NPHS2 (ab50339; Abcam), PDGFR $\beta$ (14-1402; eBiosciences), PECAM1 (553370; BD Biosciences - Pharmingen), and F4/80 (14-4801; eBiosciences), and detected with species-specific secondary antibodies coupled to Alexa Fluor 555 (Invitrogen) or DyLight 649 (Jackson ImmunoResearch) for 2 hours at room temperature. All sections were stained with Hoechst 33342 (Invitrogen) prior to mounting with ImmuMount (Fisher Scientific). Fluorescent images were acquired on a Zeiss LSM700 or LSM710 inverted confocal microscope. 
Immunohistochemistry. Immunohistochemical staining was performed on 5 - $\mu \mathrm{m}$-thick paraffin sections either after dewaxing, rehydration, proteinase $\mathrm{K}$-mediated antigen retrieval $(10 \mu \mathrm{g} / \mathrm{ml}, 5$ minutes $)$, and quenching of peroxidase activity $\left(3 \% \mathrm{H}_{2} \mathrm{O}_{2}, 5\right.$ minutes), or following section in situ hybridization according to a protocol available online (Little Group Protocols, GUDMAP; www.gudmap.org). Briefly, after blocking, the sections were incubated overnight at $4^{\circ} \mathrm{C}$ with primary antibody and then for 1 hour at room temperature. Primary antibodies used were LTL (FL-1321; Vector Labs), PECAM1 (BD Biosciences - Pharmingen; 553370), F4/80 (14-4801; eBiosciences), and LY-6G (550291; BD Biosciences - Pharmingen). The next day, sections were incubated with a species-specific, biotinylated secondary antibody prior to application of VECTASTAIN ABC reagent (Vector Laboratories) for 30 minutes at room temperature. Binding of antibodies was visualized with ImmPACT DAB peroxidase substrate solution (Vector Laboratories). Sections were postfixed in $4 \%$ paraformaldehyde prior to mounting with Immu-Mount (Fisher Scientific) and imaged on a Nikon Eclipse $90 i$.

RNA section in situ bybridization. Kidneys were perfused with icecold PBS and embedded in paraffin after overnight fixation in $4 \%$ paraformaldehyde at $4{ }^{\circ} \mathrm{C}$. Paraffin blocks were sectioned at a thickness of $5 \mu \mathrm{m}$ and in situ hybridization was performed using digoxigeninlabeled (DIG-labeled) antisense riboprobes according to a previously published protocol that had been adapted for manual processing (62). Briefly, following dewaxing and rehydration, sections were fixed in $4 \%$ paraformaldehyde, permeabilized with proteinase $\mathrm{K}(10 \mu \mathrm{g} / \mathrm{ml})$ for 20 minutes, postfixed, and acetylated $(0.375 \%$ acetic anhydride). Sections were hybridized overnight at $68^{\circ} \mathrm{C}$ with $0.5 \mu \mathrm{g} / \mathrm{ml}$ of riboprobe in hybridization buffer $(50 \%$ formamide, $5 \times$ SSC, $1 \%$ SDS, $50 \mu \mathrm{g} / \mathrm{ml}$ yeast tRNA, $50 \mu \mathrm{g} / \mathrm{ml}$ heparin). After the first posthybridization wash, sections were treated with $2 \mu \mathrm{g} / \mathrm{ml}$ RNase for 15 minutes at $37^{\circ} \mathrm{C}$. Following incubation with anti-DIG-AP antibody $\left(1: 4,000,11093274910\right.$; Roche) at $4^{\circ} \mathrm{C}$ overnight, chromogenic substrate (BM Purple; Roche) was used to visualize signals. Once a sufficient staining intensity was reached, sections were fixed in $4 \%$ paraformaldehyde and mounted with Glycergel (Dako).
Sections were imaged on a Zeiss AxioImager Z2 or a Nikon Eclipse $90 i$. Hybridization for each of the selected genes was repeated a minimum of 5 times, and all replicates gave similar staining results. No staining was observed in no-probe and no-antibody control experiments (Supplemental Figure 10 and data not shown).

Statistics. Values are represented as mean + SEM or \pm SEM. The significance of difference among groups was examined using ANOVA. A $P$ value of less than 0.05 was considered significant.

Study approval. All surgical procedures, and all mouse handling and husbandry were performed according to guidelines issued by the Institutional Animal Care and Use Committees (IACUC) at Harvard University and at the University of Southern California, and were performed after approval from each institution's IACUC committee.

\section{Acknowledgments}

This research was supported by funds from the Harvard Stem Cell Institute and the California Institute of Regenerative Medicine (53-5178-7980) to A.P. McMahon. I. Grgic was supported by a fellowship from the Deutsche Forschungsgemeinschaft (GR 3301/4-1). The authors wish to thank members of the Harvard Genome Modification Facility for their technical assistance; Fan Gao, Yibu Chen, and Meng Li at University of Southern California for their bioinformatics assistance; and the Washington University George M. O’Brien Center for Kidney Disease Research for creatinine assays.

Received for publication July 15, 2013, and accepted in revised form December 5, 2013.

Address correspondence to: Andrew P. McMahon, Department of Stem Cell Biology and Regenerative Medicine, Broad-CIRM Center, Keck School of Medicine, University of Southern California, 1425 San Pablo Street, Los Angeles, California 90089, USA. Phone: 323.442.3056; Fax: 323.442.8024; E-mail: amcmahon@med.usc.edu.
1. Warnock DG. Towards a definition and classification of acute kidney injury. J Am Soc Nephrol. 2005; 16(11):3149-3150.

2. Waikar SS, Liu KD, Chertow GM. Diagnosis, epidemiology outcomes of acute kidney injury. Clin J Am Soc Nephrol. 2008;3(3):844-861.

3. Shusterman N, Strom BL, Murray TG, Morrison G, West SL, Maislin G. Risk factors and outcome of hospital-acquired acute renal failure. Clinical epidemiologic study. Am J Med. 1987;83(1):65-71.

4. Chertow GM, Burdick E, Honour M, Bonventre JV, Bates DW. Acute kidney injury, mortality, length of stay, and costs in hospitalized patients. J Am Soc Nephrol. 2005;16(11):3365-3370.

5. Devarajan P. Update on mechanisms of ischemic acute kidney injury. J Am Soc Nephrol. 2006; 17(6):1503-1520.

6. Bonventre JV, Yang L. Cellular pathophysiology of ischemic acute kidney injury. J Clin Invest. 2011;121(11):4210-4221.

7. Bellomo R, Kellum JA, Ronco C. Defining acute renal failure: physiological principles. Intensive Care Med. 2004;30(1):33-37.

8. Humphreys BD, et al. Intrinsic epithelial cells repair the kidney after injury. Cell Stem Cell. 2008; 2(3):284-291.

9. Chawla LS, Kimmel PL. Acute kidney injury and chronic kidney disease: an integrated clinical syndrome. Kidney Int. 2012;82(5):516-524.

10. Kim TM, Ramírez V, Barrera-Chimal J, Bobadilla NA, Park PJ, Vaidya VS. Gene expression analysis reveals the cell cycle and kinetochore genes participating in ischemia reperfusion injury and early development in kidney. PLoS One. 2011;6(9):e25679.

11. Yuen PS, Jo SK, Holly MK, Hu X, Star RA. Ischemic and nephrotoxic acute renal failure are distinguished by their broad transcriptomic responses. Physiol Genomics. 2006;25(3):375-386.

12. Supavekin S, Zhang W, Kucherlapati R, Kaskel FJ, Moore LC, Devarajan P. Differential gene expression following early renal ischemia/reperfusion. Kidney Int. 2003;63(5):1714-1724.

13. Kieran NE, et al. Modification of the transcriptomic response to renal ischemia/reperfusion injury by lipoxin analog. Kidney Int. 2003;64(2):480-492.

14. Yoshida T, Tang SS, Hsiao LL, Jensen RV, Ingelfinger JR, Gullans SR. Global analysis of gene expression in renal ischemia-reperfusion in the mouse. Biochem Biophys Res Commun. 2002;291(4):787-794.

15. Yoshida $\mathrm{T}$, et al. Monitoring changes in gene expression in renal ischemia-reperfusion in the rat. Kidney Int. 2002;61(5):1646-1654.

16. Vaidya VS, Ferguson MA, Bonventre JV. Biomarkers of acute kidney injury. Annu Rev Pharmacol Toxicol. 2008;48:463-493.

17. Ko GJ, et al. Transcriptional analysis of kidneys during repair from AKI reveals possible roles for NGAL and KIM-1 as biomarkers of AKI-toCKD transition. Am J Physiol Renal Physiol. 2010; 298(6):F1472-F1483.

18. Paragas N, et al. The Ngal reporter mouse detects the response of the kidney to injury in real time. Nat Med. 2011;17(2):216-222.

19. Heiman M, et al. Development of a BACarray translational profiling approach for the molecular characterization of CNS cell types. Cell. 2008;
135(4):738-748.

20. Doyle JP, et al. Application of a translational profiling approach for the comparative analysis of CNS cell types. Cell. 2008;135(4):749-762.

21. Thomas A, et al. A versatile method for cell-specific profiling of translated mRNAs in Drosophila. PLoS One. 2012;7(7):e40276.

22. Watson FL, Mills EA, Wang X, Guo C, Chen DF, Armstrong NM. Cell type-specific translational profiling in the Xenopus laevis retina. Dev Dyn. 2012;241(12):1960-1972.

23. Tryon RC, Pisat N, Johnson SL, Dougherty JD. Development of translating ribosome affinity purification for zebrafish. Genesis. 2013;51(3):187-192.

24. Kobayashi A, et al. Six 2 defines regulates a multipotent self-renewing nephron progenitor population throughout mammalian kidney development. Cell Stem Cell. 2008;3(2):169-181.

25. Humphreys BD, et al. Fate tracing reveals the pericyte and not epithelial origin of myofibroblasts in kidney fibrosis. Am J Pathol. 2010;176(1):85-97.

26. Alva JA, et al. VE-Cadherin-Cre-recombinase transgenic mouse: A tool for lineage analysis and gene deletion in endothelial cells. Dev Dyn. 2006; 235(3):759-767.

27. Clausen BE, Burkhardt C, Reith W, Renkawitz $\mathrm{R}$, Förster I. Conditional gene targeting in macrophages and granulocytes using LysMcre mice. Transgenic Res. 1999;8(4):265-277.

28. Kiss E, et al. Peroxisome proliferator-activated receptor (PPAR)gamma can inhibit chronic renal allograft damage. Am J Pathol. 2010;176(5):2150-2162.

29. Balkovetz DF. Tight junction claudins and the kid- 
ney in sickness and in health. Biochim Biophys Acta. 2009;1788(4):858-863.

30. Duarte S, Hamada T, Kuriyama N, Busuttil RW, Coito AJ. TIMP-1 deficiency leads to lethal partial hepatic ischemia reperfusion injury. Hepatology. 2012;56(3):1074-1085.

31. Fabian SL, et al. Hedgehog-Gli pathway activation during kidney fibrosis. Am J Pathol. 2012; 180(4):1441-1453.

32. Astorga J, Carlsson P. Hedgehog induction of murine vasculogenesis is mediated by Foxf1 and Bmp4. Development. 2007;134(20):3753-3761.

33. Salehi-Tabar R, et al. Vitamin D receptor as a master regulator of the c-MYC/MXD1 network. Proc Natl Acad Sci U S A. 2012;109(46):18827-18832.

34. Ayer DE, Eisenman RN. A switch from Myc:Max to Mad:Max heterocomplexes accompanies monocyte/macrophage differentiation. Genes Dev. 1993; 7(11):2110-2119.

35. Larsson LG, Pettersson M, Oberg F, Nilsson K, Lüscher B. Expression of mad, mxi1, max c-myc during induced differentiation of hematopoietic cells: opposite regulation of mad c-myc. Oncogene. 1994;9(4):1247-1252.

36. Khairoun $M$, et al. Renal ischemia-reperfusion induces a dysbalance of angiopoietins, accompanied by proliferation of pericytes and fibrosis. Am J Physiol Renal Physiol. 2013;305(6):F901-F910

37. de Vries DK, et al. Renal ischemia-reperfusion induces release of angiopoietin-2 from human grafts of living deceased donors. Transplantation. 2013; 96(3):282-289.

38. Schioppa T, et al. Regulation of the chemokine receptor CXCR4 by hypoxia. J Exp Med. 2003; 198(9):1391-1402.

39. Schutyser E, et al. Hypoxia enhances CXCR4 expression in human microvascular endothelial cells human melanoma cells. Eur Cytokine Netw. 2007;18(2):59-70.

40. Strasser GA, Kaminker JS, Tessier-Lavigne M. Microarray analysis of retinal endothelial tip cells identifies CXCR4 as a mediator of tip cell morphol- ogy branching. Blood. 2010;115(24):5102-5110

41. Zünd $G$, et al. Hypoxia enhances induction of endothelial ICAM-1: role for metabolic acidosis proteasomes. Am J Physiol. 1997; 273(5 pt 1):C1571-C1580.

42. Hess DC, Zhao W, Carroll J, McEachin M, Buchanan $\mathrm{K}$. Increased expression of ICAM-1 during reoxygenation in brain endothelial cells. Stroke. 1994;25(7):1463-1467.

43. Kelly KJ, et al. Intercellular adhesion molecule1 -deficient mice are protected against ischemic renal injury. J Clin Invest. 1996;97(4):1056-1063.

44. Guerrot D, Dussaule JC, Kavvadas P, Boffa JJ, Chadjichristos CE, Chatziantoniou C. Progression of renal fibrosis: the underestimated role of endothelial alterations. Fibrogenesis Tissue Repair. 2012;5(suppl 1):S15.

45. Gardiner NJ, Moffatt S, Fernyhough P, Humphries MJ, Streuli CH, Tomlinson DR. Preconditioning injury-induced neurite outgrowth of adult rat sensory neurons on fibronectin is mediated by mobilisation of axonal alpha5 integrin. Mol Cell Neurosci. 2007;35(2):249-260.

46. Srouji S, et al. Lentiviral-mediated integrin $\alpha 5$ expression in human adult mesenchymal stromal cells promotes bone repair in mouse cranial longbone defects. Hum Gene Ther. 2012;23(2):167-172.

47. Jin J, et al. Soluble FLT1 binds lipid microdomains in podocytes to control cell morphology glomerular barrier function. Cell. 2012;151(2):384-399.

48. Basile DP, Sutton TA. Activated pericytes and the inhibition of renal vascular stability: obstacles for kidney repair. J Am Soc Nephrol. 2012;23(5):767-769.

49. Basile DP. Rarefaction of peritubular capillaries following ischemic acute renal failure: a potential factor predisposing to progressive nephropathy. Curr Opin Nephrol Hypertens. 2004;13(1):1-7.

50. Leonard EC, Friedrich JL, Basile DP. VEGF-121 preserves renal microvessel structure ameliorates secondary renal disease following acute kidney injury. Am J Physiol Renal Physiol. 2008;295(6):F1648-F1657.

51. Rovida E, et al. ERK5/BMK1 is indispensable for optimal colony-stimulating factor 1 (CSF-1)-induced proliferation in macrophages in a Src-dependent fashion. J Immunol. 2008;180(6):4166-4172.

52. Lochhead PA, Gilley R, Cook SJ. ERK5 and its role in tumour development. Biochem Soc Trans. 2012;40(1):251-256.

53. Wang X, Tournier C. Regulation of cellular functions by the ERK5 signalling pathway. Cell Signal. 2006;18(6):753-760

54. Tsanov KM, Nishi Y, Peterson KA, Liu J, Baetscher M, McMahon AP. An embryonic stem cell-based system for rapid analysis of transcriptional enhancers. Genesis. 2012;50(5):443-450.

55. Aker M, et al. Extended core sequences from the cHS4 insulator are necessary for protecting retroviral vectors from silencing position effects. Hum Gene Ther. 2007;18(4):333-343.

56. Kobayashi A, et al. Six 2 defines regulates a multipotent self-renewing nephron progenitor population throughout mammalian kidney development. Cell Stem Cell. 2008;3(2):169-181.

57 . Humphreys BD, et al. Fate tracing reveals the pericyte and not epithelial origin of myofibroblasts in kidney fibrosis. Am J Pathol. 2010;176(1):85-97.

58. Alva JA, et al. VE-Cadherin-Cre-recombinase transgenic mouse: a tool for lineage analysis and gene deletion in endothelial cells. Dev Dyn. 2006; 235(3):759-767.

59. Clausen BE, Burkhardt C, Reith W, Renkawitz R, Förster I. Conditional gene targeting in macrophages and granulocytes using LysMcre mice. Transgenic Res. 1999;8(4):265-277.

60. Weight SC, Furness PN, Bell PR, Nicholson ML. A new model of renal warm ischemia reperfusion injury. Transplant Proc. 1997;29(7):3002-3003.

61. Kennedy SE, Erlich JH. Murine renal ischaemia-reperfusion injury. Nephrology (Carlton). 2008;13(5):390-396.

62. Rumballe B, Georgas K, Little MH. High-throughput paraffin section in situ hybridization and dual immunohistochemistry on mouse tissues. CSH Protoc. 2008;2008:pdb.prot5030. 